\title{
Public microarray repository semantic annotation with ontologies employing text mining and expression profile correlation David Ruau*1, Corinna Kolárik ${ }^{2,3}$, Heinz-Theodor Mevissen², Emmanuel Müller ${ }^{4}$, Ira Assent ${ }^{4}$, Ralph Krieger ${ }^{4}$, Thomas Seidl ${ }^{4}$, Martin Hofmann-Apitius ${ }^{2,3}$ and Martin Zenke ${ }^{1,5}$
}

\author{
Address: ${ }^{1}$ Department of Cell Biology, Institute for Biomedical Engineering, RWTH Aachen, University Medical School, 52074 Aachen, Germany, \\ ${ }^{2}$ Fraunhofer Institute SCAI, Schloss Birlinghoven, 53754 Sankt Augustin, Germany, ${ }^{3}$ Bonn-Aachen International Center for Information \\ Technology (B-IT), Department of Applied Life Science Informatics, 53113 Bonn, Germany, ${ }^{4}$ Data management and data exploration group, \\ RWTH Aachen University, Germany and ${ }^{5}$ Helmholtz Institute for Biomedical Engineering, RWTH Aachen University, 52074 Aachen, Germany \\ Email: David Ruau* - David.Ruau@rwth-aachen.de \\ * Corresponding author
}

from Fourth International Society for Computational Biology (ISCB) Student Council Symposium

Toronto, Canada. 18 July 2008

Published: 30 October 2008

BMC Bioinformatics 2008, 9(Suppl I0):O5 doi:10.1 186/I47|-2105-9-SI0-O5

This abstract is available from: http://www.biomedcentral.com//47/-2/05/9/SI0/O5

(c) 2008 Ruau et al; licensee BioMed Central Ltd

\section{Public microarray repository annotation}

Gene Expression Omnibus (GEO) [1] is the largest public web repository of microarray experiments. GEO, like ArrayExpress and Stanford MicroArray Database, provides descriptions of microarray experiments in free text making it difficult to search and comprehensively link those data to other knowledge resources. Text mining techniques applied to microarray experiment annotation are challenged by poor and/or ambiguous free text description and consequently leave some objects unlabelled. Previous work organized GEO entries at the level of series (GSE) and data sets (GDS) [2] using the Unified Medical Language System (UMLS) [3]. GSE and GDS description are often too broad and a better quality of annotation can be achieved if the GEO samples (GSM) are considered directly. Here we report on a novel approach for annotating GSM objects by employing a combination of text mining and global gene expression similarity. We hypothesize that the biological material analyzed on microarrays is related if unlabeled and labeled objects are highly similar in expression values and hence the class/annotation of one object can help annotate an unlabeled object. Our new method allows us to achieve a higher percentage of semantic annotation by combining both types of information stored in microarray databases.

\section{Results}

The GSM free text description (downloaded from GEO in November 2007) was mined using ProMiner [4], a software for Named Entity Recognition based on dictionaries of cell, tissue and disease ontologies from OBO [5] plus cell line resources. This resulted in 73.5-97.6\% class labeling of the GSM objects (Table 1). Next the labeled objects were used to annotate the unlabeled objects. We computed the correlation matrix for all the objects where the raw data were available and followed the nearest neighbor approach [6] to identify the nearest labeled object within a $\delta$ range. The $\delta$ value is an input parameter determined empirically and limits the propagation of too dissimilar annotations. In this study we selected a delta value of 0.04 and observed an increase of the annotation percentage up to $4.9 \%$, depending on the platform. The class labeling overall percentage after annotation propagation reached $78.4-99.4 \%$ (Table 1). The results were then stored into a relational database allowing to semantically search for microarray experiments.

\section{Conclusions and perspectives}

The class/annotation propagation from a labeled object to an unlabeled object works only if there is one labeled object within the $\delta$ range. Thus the chances of class prop- 
Table I: GSM object annotation coverage.

\begin{tabular}{lcccc}
\hline Platform & GSM object number (a) & Labeled objects by ProMiner (b) & $\begin{array}{c}\text { Raw data available/ProMiner } \\
\text { labeled (c) }\end{array}$ & Propagated annotation (d) \\
\hline mouse4302 (GPLI26I) & 4288 & $87.2 \%$ & $3362 / 2852$ & $145[90.6 \%]$ \\
mouse430a2 (GPL339) & 3521 & $91.1 \%$ & $321 / 288$ & $25[91.8 \%]$ \\
moe430b (GPL340) & 795 & $97.6 \%$ & $545 / 524$ & $14[99.4 \%]$ \\
mgu74av2 (GPL8I) & 4676 & $86.2 \%$ & $2390 / 2120$ & $183[89.5 \%]$ \\
Hgu95av2 (GPL9I) & 4512 & $73.5 \%$ & $1865 / 1325$ & $221[78.4 \%]$ \\
\hline
\end{tabular}

We performed our study on a subset of platforms from Affymetrix. (a) Total number of GSM objects present in GEO per platform (November 2007). (b) Percentage of objects labeled by ProMiner. (c) Objects with raw data stored in GEO and number of labeled objects with raw data. (d) Number of propagated annotations and final percentage of labeled objects

agation increase with the number of available objects. We plan to improve on this by merging different types of microarray platforms by using tools like AILUN [7] as well as adding a confidence score to the propagated annotations. Ultimately, the annotation process will be automatized and the resulting database made freely available.

\section{References}

I. Gene Expression Omnibus [http://www.ncbi.nlm.nih.gov/geo/]

2. Butte AJ, Kohane IS: Creation and implications of a phenomegenome network. Nat Biotechnol 2006, 24:55-62.

3. Unified Medical Language System [http://www.nlm.nih.gov/ research/umls/]

4. Hanisch D, Fundel K, Mevissen HT, Zimmer R, Fluck J: ProMiner: rule-based protein and gene entity recognition. $B M C$ Bioinformatics 2005, 6(SuppI I):SI4.

5. The Open Biomedical Ontologies [http://obofoundry.org/]

6. Dasarathy BV: Nearest Neighbor (NN) Norms: NN Pattern Classification Techniques IEEE Computer Society Press; 199I.

7. Chen R, Li L, Butte AJ: AlLUN: reannotating gene expression data automatically. Nature Methods 2007, 4:879.

Publish with Bio Med Central and every scientist can read your work free of charge

"BioMed Central will be the most significant development for disseminating the results of biomedical research in our lifetime. "

Sir Paul Nurse, Cancer Research UK

Your research papers will be:

- available free of charge to the entire biomedical community

- peer reviewed and published immediately upon acceptance

- cited in PubMed and archived on PubMed Central

- yours - you keep the copyright

Submit your manuscript here:

http://www.biomedcentral.com/info/publishing_adv.asp
BioMedcentral 